\title{
Diversidad de aves y mamíferos marinos en bahía San Pedro, costa de Purranque, centro-sur de Chile.
}

\section{Diversity of seabirds and marine mammals in bahía San Pedro, Purranque coast, south- central Chile.}

\author{
Jaime A. Cursach ${ }^{1,2^{*}}$, Jaime R. Rau ${ }^{1,2}$, Jaime Ojeda ${ }^{3}$, Jonathan Vilugrón ${ }^{1,2}$, Claudio Tobar ${ }^{1,2}$, Carlos \\ OYARZÚN ${ }^{2}$, Oscar SOTO ${ }^{4}$ \& CRISTIÁn G. SUAZO ${ }^{5}$ \\ ${ }_{1}^{1}$ Programa de Magíster en Ciencias, Escuela de Postgrado, Universidad de Los Lagos, Cochrane 1070, Osorno, Chile. \\ ${ }^{2}$ Laboratorio de Ecología \& Programa IBAM, Universidad de Los Lagos, Casilla 933, Osorno, Chile. \\ ${ }^{3}$ Programa de Magíster en Ciencias, Universidad de Magallanes, Casilla 113-D, Punta Arenas, Chile. Parque Etnobotánico \\ Omora, Puerto Williams, Chile. Instituto de Biodiversidad y Ecología (IEB), Santiago, Chile. \\ ${ }^{4}$ Laboratorio de Oceanografía, Depto. de Acuicultura y Recursos Acuáticos, Universidad de Los Lagos, Casilla 933, Osorno, \\ Chile. \\ ${ }^{5}$ Instituto de Ciencias Ecológicas y Evolutivas, Facultad de Ciencias, Universidad Austral de Chile, casilla 567, Valdivia, Chile. \\ *Email: jcurval@gmail.com
}

\begin{abstract}
RESUMEN
Entre los años 2007 y 2009, se estudió la diversidad de aves y mamíferos marinos que habitan bahía San Pedro (comuna de Purranque, provincia de Osorno). Evaluamos la composición, diversidad y abundancia de estos vertebrados, que presentó 18 especies de aves marinas, de las cuales Spheniscus magellanicus y Puffinus griseus fueron las más abundantes. Dos especies de pingüinos, una gaviota y un gaviotín se reproducen en la bahía. El ensamble de mamíferos marinos estuvo compuesto por siete especies, de las cuales Otaria flavescens y Lagenorhynchus australis fueron las más abundantes. Una especie de lobo marino y nutria de mar se reproducen en la bahía. Los resultados demuestran el potencial que posee esta bahía para la conservación de biodiversidad marina y el desarrollo del turismo de intereses especiales que promuevan la conservación. A partir de esta información, recomendamos establecer un programa de estudios a largo plazo que asesore a la comunidad local en la planificación, regulación y evaluación de las actividades de turismo a desarrollar.
\end{abstract}

Palabras claves: conservación, Huilliche, manejo costero, turismo, vertebrados marinos.

\begin{abstract}
Between 2007 and 2009, we studied the diversity of seabirds and marine mammals inhabiting San Pedro Bay (Purranque, coastal area of the Osorno province). We assessed composition, diversity and abundance of these vertebrates that included 18 species of seabirds, with Spheniscus magellanicus and Puffinus griseus as the most abundant. Two species of penguin, a gull and a tern breed in the area. Marine mammal assemblages were composed of seven species, from which Otaria flavescens and Lagenorhynchus australis were the most abundant. A species of sea lion and an otter breed in the area. These results show the potential of this area for biological conservation and the development of special interest tourism initiatives, which promote conservation. Based on this information, we recommend establishing long-term study programs that will provide guidance to the local community, in terms of planning, regulation and assessment of tourism activities that can be developed.
\end{abstract}

KEYWORDS: coastal management, conservation, Huilliche, marine vertebrates, tourism.

\section{INTRODUCCIÓN}

El borde costero norte $\left(40^{\circ} 15^{\prime}-41^{\circ} 35^{\prime} \mathrm{S}\right)$ de la Región de Los Lagos (en adelante BCNRL) en el centro-sur de Chile, se caracteriza por ser una zona poco intervenida por la acción humana, con ausencia de centros urbanos y escasos caminos para el tránsito de vehículos pesados. En suma a lo anterior, la accidentada geografía del lugar permite la existencia del último gran reducto de bosque laurifolio siempreverde en Sudamérica, comúnmente conocido como Selva Valdiviana (Smith-Ramírez 2004), conformando un paisaje único de bosque nativo y mar. Estas características 
han promovido que organismos públicos y privados generen propuestas para transformar esta zona en una reserva de uso turístico. Ante ello y pese a que el año 2005 se estableció en dicho borde costero el Área Marina y Costera Protegida de Múltiples Usos Lafken-Mapulahual (40³7'-4048'S) existe poca información publicada sobre los componentes de este ambiente. Por ello, se hace urgente desarrollar estudios sobre la biodiversidad presente en este ecosistema.

Los escasos registros publicados indican la presencia de sitios reproductivos de aves y mamíferos marinos en la zona (Córdova et al. 2009; Cursach et al. 2009). Las colonias de aves y mamíferos marinos son particularmente vulnerables a las actividades humanas (e.g. turismo de masas), en donde la perturbación antrópica se transforma en fuente de estrés conductual y fisiológico que altera el éxito reproductivo de dicha fauna (Ellenberg et al. 2006; Constantine 1999; Fowler 1999). Pese a ello, existen programas de turismo en conservación (e.g. turismo de intereses especiales, turismo científico, ecoturismo) que al ser planificados y regulados han logrado ser sustentables en el tiempo ( $c f$. Skewgar et al. 2009; Yorio et al. 2001). Esta actividad productiva puede llegar a ser una buena alternativa para el desarrollo sustentable de las comunidades rurales y originarias que habitan el BCNRL.

Para diseñar e implementar estrategias sustentables de desarrollo en el BCNRL, es necesario contar con información ecológica básica sobre los componentes de este ecosistema. Por ello, el objetivo del presente trabajo fue determinar la composición, diversidad y abundancia de las poblaciones de aves y mamíferos marinos presentes en bahía San Pedro, localidad ubicada en el centro latitudinal del BCNRL.

\section{MATERIALES Y MÉTODOS}

\section{Área de ESTUdio}

Bahía San Pedro (4056’30,47"S; 7351'48,29"O) se ubica en la costa de la comuna de Purranque, provincia de Osorno, Región de Los Lagos, centro-sur de Chile (Fig. 1). Dentro de esta bahía se encuentra caleta San Pedro, asentamiento humano constituido por no más de 100 personas, cuyas actividades productivas se sustentan de la pesca artesanal (Área de manejo de recursos bentónicos) y la extracción de tejuelas de alerce Fitzroya cupressoides [(Molina) Johnston]. En la zona sur-oeste de esta bahía, a una distancia de 3,5 km de la costa, existe un sistema de islotes ( 8 ha) compuesto por tres grandes promontorios rocosos cubiertos por vegetación de tipo arbustiva con predominio de chupallilla (Bromelia sp.). A lo largo de la costa oriental del sistema de islotes existen extensas y abundantes formaciones boscosas del alga Macrocystis pyrifera [(L.) C. Agardh]. La temperatura y salinidad superficial del agua de mar en bahía San Pedro, durante invierno 2007 fue de $8,8^{\circ} \mathrm{C}$ y 34,3 psu respectivamente, mientras que en verano 2008 fue de 12,1 ${ }^{\circ} \mathrm{C}$ y 36,4 psu.

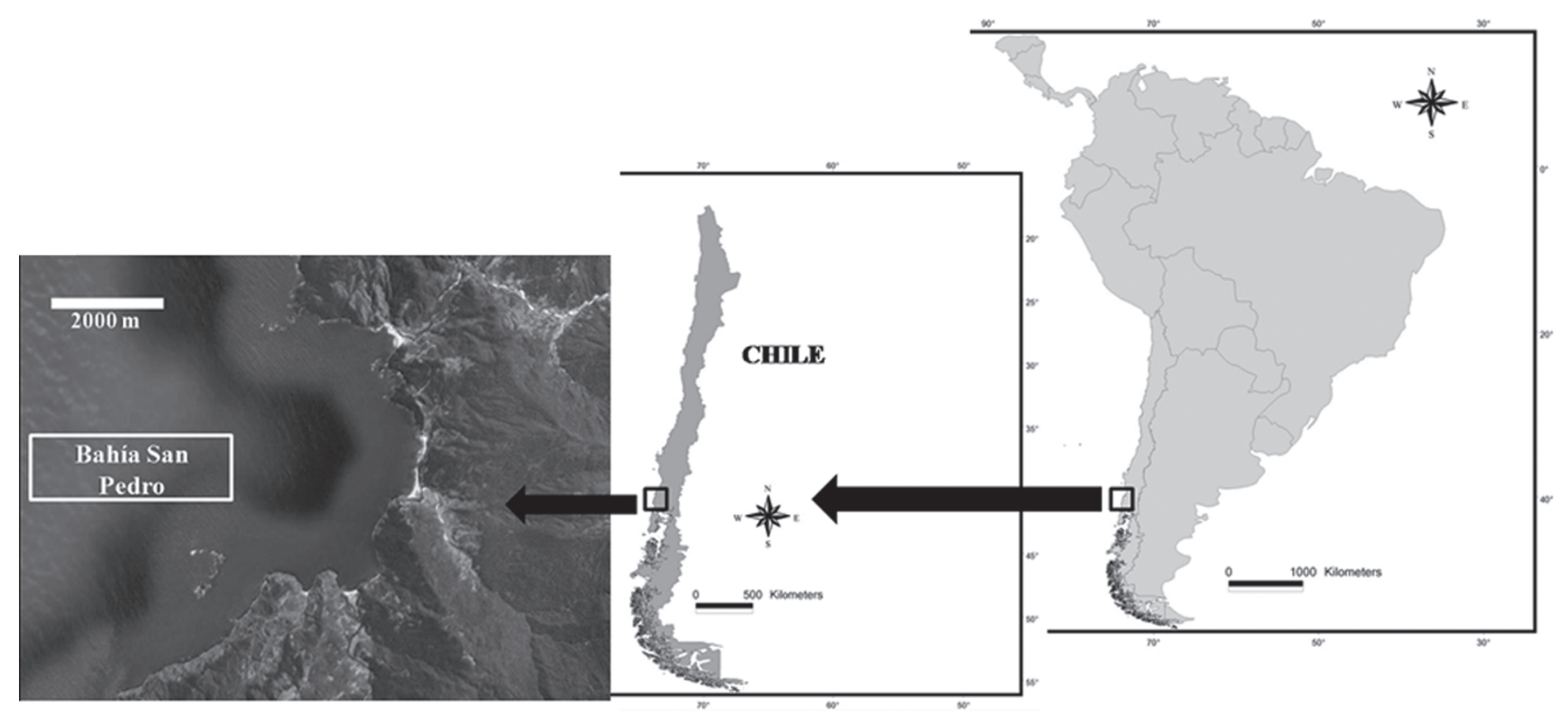

Figura 1. Ubicación geográfica de Bahía San Pedro, costa de Purranque, sur de Chile.

FIgURE 1. Geographic location of San Pedro Bay, in the coastal area of Purranque, southern Chile. 
COMPOSICIÓN, DIVERSIDAD Y ABUNDANCIA

Durante los meses de enero, febrero (verano), septiembre, octubre y diciembre (primavera) de 2007, enero (verano) 2008 y enero (verano) 2009, se realizaron censos y monitoreos de las especies de aves y mamíferos marinos presentes en esta bahía. Debido a limitaciones logísticas y de seguridad, solo se logró censar a las aves nidificantes en la porción norte del sistema de islotes (i.e. islote Pingüinos), el cual posee un área de 2,5 ha, pendiente de $45^{\circ}$ y una altitud máxima de 35 m.s.n.m.

La abundancia de aves marinas se determinó mediante transectos de navegación desde caleta San Pedro hasta el sistema de islotes, abarcando una distancia de $3 \mathrm{~km}$ $\mathrm{y}$ totalizando 14 transectos durante todo el periodo de estudio. En cada uno de estos transectos se registró la riqueza y abundancia de las aves marinas observadas en vuelo y posadas sobre el mar, dentro de una distancia de $150 \mathrm{~m}$ a ambos lados de la embarcación (bote de $6 \mathrm{~m}$ de eslora con motor fuera de borda de $45 \mathrm{hp}$ ), registrando la abundancia de las aves cada 10 minutos, según lo propuesto por Tasker et al. (1984). Mediante caminata silenciosa se recorrió la totalidad del islote Pingüinos (transecto de 0,5 $\mathrm{km}$ ), realizando conteos directos de las aves presentes. Las poblaciones de aves marinas nidificantes también fueron evaluadas por conteo directo de los nidos aparentemente ocupados, los que se definieron como nidos construidos con capacidad para mantener los huevos y ocupados como mínimo por una especie de ave (Bibby et al. 2000). Las observaciones fueron realizadas de manera simultánea por dos observadores, entre las 8:00 y 13:00 h, totalizando $180 \mathrm{~h}$ de observación durante todo el período de estudio.

Para registrar la riqueza y abundancia de mamíferos marinos, se establecieron cuatro sitios de observación seleccionados por su accesibilidad y visibilidad desde el mirador de caleta San Pedro hacia toda la bahía. Desde estos puntos se realizaron conteos directos y simultáneos por cuatro observadores. Mediante los mismos transectos de navegación utilizados para censar aves marinas, dos observadores identificaron y cuantificaron la abundancia de mamíferos marinos desde la proa de la embarcación, realizando desvíos en la ruta de navegación para acercarse a los animales y garantizar su correcta identificación. Para registrar las poblaciones reproductivas de mamíferos marinos presentes en el sistema de islotes, se establecieron dos sitios de observación ubicados en los puntos más altos del islote Pingüinos, desde los cuales se realizaron conteos directos y simultáneos por dos observadores. Todos los censos realizados a las poblaciones de mamíferos marinos presentes en la bahía fueron ejecutados entre las 8:00 y 17:00 $\mathrm{h}$, totalizando $210 \mathrm{~h}$ de observación durante todo el período de estudio. Esfuerzo de observación similar al utilizado por especialistas en el sur del país (Aguayo et al. 2006).
ANÁLISIS DE LOS DATOS

Expresamos la riqueza de especies (S) simplemente como el número total de especies identificadas. Sin embargo, para comparar las riquezas de especies de aves y mamíferos marinos utilizamos el método de Helsche \& Forrester (1985) el que, a través del acuchillamiento ("jackknife") de los datos originales, genera pseudovalores de tendencia central y de dispersión que permiten la docimación de hipótesis estadísticas. Para su cálculo empleamos el programa computacional provisto en Krebs (1999). Este programa considera como entrada (“input") una matriz rectangular de datos de presencia y ausencia (i.e. incidencias de especies) conformada por cuadrantes de muestreo (columnas) y especies (filas). Para ello deben existir en los muestreos especies "únicas". Es decir, aquellas que estuvieron presentes solo en uno de los muestreos. Como cuadrantes de muestreo utilizamos nuestros resultados obtenidos para cada uno de los cuatro censos estacionales realizados. Como salida (“output") el programa entrega la riqueza promedio acuchillada, la desviación estándar (de la cual se obtiene el error estándar dividiéndola por la raíz cuadrada del tamaño de la muestra, $c f$. Zar 1974) y los intervalos de confianza para un $95 \%$ de confiabilidad. Así, dos riquezas promedio acuchilladas y sus errores estándar asociados pueden compararse mediante una prueba $\mathrm{t}$ para dos muestras independientes (Zar 1974).

También utilizamos la función de acumulación de especies no asintótica de Clench (Soberón \& Llorente 1993). Esta se obtiene de dividir el intercepto a per la pendiente b y asume que el número de especies nuevas a descubrir en un muestreo aumenta con la experiencia del observador. Para ello se hace una regresión no lineal entre los muestreos (variable independiente) versus el número de especies acumuladas (variable dependiente). Utilizamos para estos efectos el programa CURVE EXPERT. Como una medida de la bondad de cada modelo este programa utiliza el coeficiente de correlación producto-momento y el error estándar.

Por último, para obtener la diversidad ecológica se utilizó el índice $\mathrm{H}^{\prime}$ de Shannon. Mediante el programa FRANJA (Franja 1993), paquete estadístico especializado para cálculos de diversidad biológica, se obtuvo su valor en logaritmos naturales con su varianza analítica asociada. Asumiendo que las estimaciones de abundancia y diversidad minimizan la biodiversidad esperada, para el cálculo de la diversidad ecológica utilizamos el número máximo de individuos detectados de aves y mamíferos marinos en los muestreos realizados. De esta manera dos índices de diversidad y sus varianzas pueden compararse estadísticamente con la prueba de Hutcheson (1970), que es análoga a una prueba $\mathrm{t}$ de Student (Zar 1974). 
Para conocer la diversidad beta temporal o recambio de especies en bahía San Pedro, se compararon los muestreos estacionales tanto de aves como de mamíferos marinos a través del porcentaje de similitud SIMPER. Para este objetivo se transformaron los datos de avistamientos de aves y mamíferos marinos en el área de estudio, ya que estos presentaban valores muy altos y muy bajos, utilizando la transformación raíz cuarta. Para el porcentaje de similitud SIMPER se utilizó el programa computacional PRIMER. Se calculó la diversidad beta tanto para aves como para mamíferos marinos mediante el índice de reemplazo de especies de Whittaker (Moreno 2001).

Para identificar el estado de conservación de las aves y mamíferos marinos presentes en bahía San Pedro se utilizó el Sistema de clasificación de especies silvestres en categorías de conservación (criterio nacional) y la Lista roja de especies amenazadas de la UICN (criterio internacional).

\section{RESULTADOS}

La composición taxonómica de aves marinas se estructuró en ocho familias, representadas por 18 especies (Tabla 1). Las especies más abundantes fueron el pingüino de Magallanes Spheniscus magellanicus (Forster, 1781), la fardela negra Puffinus griseus (Gmelin, 1789) y la gaviota dominicana Larus dominicanus (Lichtenstein, 1823) (Tabla 1). Dentro de las aves nidificantes en bahía San Pedro, el pingüino de Magallanes fue la especie más abundante (Tabla 2). Mientras que la gaviota de Franklin Larus pipixcan (Wagler, 1831) fue el ave visitante con mayor abundancia en la bahía (Tabla 1).

La composición taxonómica de mamíferos marinos se estructuró en cinco familias, representadas por siete especies (Tabla 3). La especie más abundante fue el lobo marino común Otaria flavescens (Shaw, 1800), seguido por el delfín austral Lagenorhynchus australis (Peale, 1848) (Tabla 3). De manera similar a las aves marinas, en el sistema de islotes se ubicaron las poblaciones reproductivas de chungungo Lontra felina (Molina, 1782) y lobo marino común.

La riqueza de especies de aves marinas $(\mathrm{S}=18)$ superó 2,7 veces la riqueza de especies de mamíferos marinos $(S=7)$. La riqueza promedio y su error estándar de aves, estimada mediante acuchillamiento de datos [Especies únicas $=2$ : petrel gigante antártico Macronectes giganteus (Gmelin, 1789) y el piquero Sula variegata (Tschudi, 1845)] fue igual a 19,5 $\pm 0,353$ con intervalos de confianza [IC (95\%)] que variaron entre 14,7 y 24,3 . En mamíferos la riqueza [Especies únicas $=3$ : leopardo marino Hydrurga leptonyx (de Blainville, 1820), ballena azul Balaenoptera musculus (Linnaeus, 1758) y la orca Orcinus orca (Linnaeus, 1758)] fue igual a 9,3 $\pm 0,3$ con IC (95\%) que variaron entre 6,9-
11,6. Las riquezas acuchilladas difirieron estadísticamente $(\mathrm{t}=2,252, \mathrm{~g} .1 .=23, \mathrm{P}<0,05)$.

A su vez, la función de acumulación de Clench para aves marinas, estimada con el programa CURVE EXPERT (coeficiente de correlación producto-momento $r=0,941$, EE $=0,412)$ fue igual a 19,1 especies $(a=106, b=5,550)$. En el caso de los mamíferos marinos, el modelo de regresión no lineal presentó valores para $\mathrm{r}=0,955$ y $\mathrm{EE}=0,683$ y la función de acumulación fue igual a 12,0 especies $(\mathrm{a}=5,119$, $b=0,437)$.

El mismo patrón encontrado para la riqueza de especies se repitió en relación con la diversidad ecológica promedio y su varianza analítica asociada $\left(\mathrm{H}^{\prime} \pm \mathrm{s}^{2} \mathrm{H}^{\prime}\right)$, la que para las aves marinas fue igual a 2,057 $\pm 0,0001 \mathrm{y}$ para los mamíferos marinos $0,580 \pm 0,005$. Esta diferencia de 3,5 veces fue estadísticamente significativa (prueba de Hutcheson $\mathrm{t}=$ 20,803; $\mathrm{P}<0,05)$.

Para ensamble de aves marinas se observó que las temporadas con mayor similitud fueron los veranos de 2008 y 2009 (promedio disimilitud $=8,62$ ), seguido por los veranos de 2007 y 2008 (promedio disimilitud $=9,05$ ) y por los veranos de 2007 y 2009 (promedio disimilitud = 10,18). La mayor disimilitud se encontró en la primavera de 2007 y el verano de 2007 (promedio disimilitud $=16,07$ ), señalando que las aves avistadas en esas temporadas son las que mayor difieren en especies y su abundancia comparadas con los otros años muestreados. Para el ensamble de mamíferos marinos se observó que las temporadas con mayor similitud fueron la primavera de 2007 y el verano de 2009 (promedio disimilitud $=17,61)$. La mayor disimilitud se encontró durante el verano de 2007 y el verano de 2008 (promedio disimilitud $=26,30$ ), señalando que los mamíferos marinos avistados en esas temporadas son los que mas difieren en especies y en abundancia comparados con los otros años muestreados.

El cálculo del índice de Whittaker indicó que el valor de diversidad beta en aves marinas fue mayor en la primavera de $2007(1,16)$, señalando que en esa temporada el recambio de especies fue mayor que en todos los veranos muestreados. Mientras que en los mamíferos marinos se observó que durante el verano de 2007 se obtuvo el menor valor de diversidad beta de entre todas las temporadas muestreadas, señalando que en dicha temporada se produjo el menor recambio de especies.

En relación a los estados de conservación de las aves y mamíferos marinos presentes en bahía San Pedro, los mamíferos presentan mayores $(71,4 \%)$ problemas de conservación que las aves $(33,3 \%)$, con una diferencia de $38,1 \%$ (Tablas 1 y 3 ). 
TABla 1. Listado de la riqueza de especies y abundancia estacional del ensamble de aves presente en bahía San Pedro, centro-sur de Chile (+ = y más individuos). Estado de conservación de cada especie (E.C.) según criterios (nacional / internacional), en donde N: no indica, MP: menor preocupación, IC: insuficientemente conocida, CA: casi amenazado, V: vulnerable, EP: en peligro de extinción.

TABLE 1. List of species richness and seasonal abundance of birds assemblage present in bahía San Pedro, south-central Chile (+= and more individuals). Conservation status of each species (EC) according to criteria (national / international), where N: not indicated, MP: least concern, IC: insufficiently known, CA: near threatened, V: vulnerable, EP: endangered of extinction.

\begin{tabular}{|c|c|c|c|c|c|}
\hline Clasificación taxonómica & Ver - 07 & Prim - 07 & Ver - 08 & Ver - 09 & E.C. \\
\hline \multicolumn{6}{|l|}{ Procellariidae } \\
\hline Macronectes giganteus & 0 & 1 & 0 & 0 & $\mathrm{~N} / \mathrm{MP}$ \\
\hline Puffinus creatopus & $20+$ & $50+$ & $20+$ & $20+$ & $\mathrm{EP} / \mathrm{V}$ \\
\hline Puffinus griseus & $100+$ & $500+$ & $288+$ & $193+$ & $\mathrm{N} / \mathrm{CA}$ \\
\hline \multicolumn{6}{|l|}{ Spheniscidae } \\
\hline Spheniscus humboldti & 25 & 20 & 22 & 21 & $\mathrm{~V} / \mathrm{V}$ \\
\hline Spheniscus magellanicus & 118 & 139 & 176 & 184 & $\mathrm{~N} / \mathrm{CA}$ \\
\hline \multicolumn{6}{|l|}{ Sulidae } \\
\hline Sula variegata & 0 & 3 & 0 & 0 & $\mathrm{~N} / \mathrm{MP}$ \\
\hline \multicolumn{6}{|l|}{ Pelecanidae } \\
\hline Pelecanus thagus & 38 & 58 & 9 & 79 & $\mathrm{~N} / \mathrm{CA}$ \\
\hline \multicolumn{6}{|l|}{ Phalacrocoracidae } \\
\hline Phalacrocorax brasilianus & 35 & 12 & 27 & 19 & $\mathrm{~N} / \mathrm{MP}$ \\
\hline Phalacrocorax magellanicus & 5 & 7 & 6 & 6 & $\mathrm{~N} / \mathrm{MP}$ \\
\hline Phalacrocorax gaimardi & 5 & 26 & 5 & 18 & $\mathrm{~N} / \mathrm{CA}$ \\
\hline Phalacrocorax atriceps & 1 & 15 & 3 & 7 & $\mathrm{~N} / \mathrm{MP}$ \\
\hline \multicolumn{6}{|l|}{ Cathartidae } \\
\hline Coragyps atratus & 1 & 5 & 6 & 2 & $\mathrm{~N} / \mathrm{MP}$ \\
\hline \multicolumn{6}{|l|}{ Haematopodidae } \\
\hline Haematopus palliatus & 10 & 4 & 1 & 1 & $\mathrm{~N} / \mathrm{MP}$ \\
\hline Haematopus ater & 6 & 2 & 3 & 2 & $\mathrm{~N} / \mathrm{MP}$ \\
\hline \multicolumn{6}{|l|}{ Laridae } \\
\hline Larus dominicanus & 89 & 82 & 92 & 167 & $\mathrm{~N} / \mathrm{MP}$ \\
\hline Larus pipixcan & 259 & 21 & 34 & 93 & $\mathrm{~N} / \mathrm{MP}$ \\
\hline Larus maculipennis & 12 & 3 & 7 & 24 & $\mathrm{~N} / \mathrm{MP}$ \\
\hline Sterna hirundinacea & 8 & 50 & 20 & 113 & $\mathrm{~N} / \mathrm{MP}$ \\
\hline
\end{tabular}

TABLa 2. Abundancia estacional de las aves marinas nidificantes en bahía San Pedro, centro-sur de Chile (+= y más individuos).

TABLE 2. Seasonal abundance of seabird species nesting in San Pedro Bay, south-central Chile ( $+=$ and more individuals).

\begin{tabular}{lcccc}
\hline \multicolumn{1}{c}{ Especies } & Ver - 07 & Prim - 07 & Ver - 08 & Ver - 09 \\
\hline Larus dominicanus & $7+$ & $6+$ & $10+$ & $11+$ \\
Spheniscus humboldti & $3+$ & $4+$ & $4+$ & $5+$ \\
Spheniscus magellanicus & $321+$ & $231+$ & $349+$ & $353+$ \\
Sterna hirundinacea & $14+$ & $12+$ & $20+$ & $17+$ \\
\hline
\end{tabular}


TABla 3. Listado de la riqueza de especies y abundancia estacional del ensamble de mamíferos marinos presente en bahía San Pedro, sur de Chile (* = población reproductiva). Estado de conservación de cada especie (E.C.), tal como lo descrito en Tabla 1.

TABLE 3. List of species richness and seasonal abundance of marine mammal assemblages present in San Pedro Bay, southern Chile $(*=$ breeding population). Conservation status of each species (EC), such as described in Table 1.

\begin{tabular}{|c|c|c|c|c|c|}
\hline Clasificación taxonómica & Ver - 07 & Pri - 07 & Ver -08 & Ver - 09 & E.C. \\
\hline \multicolumn{6}{|l|}{ Mustelidae } \\
\hline Lontra felina * & 6 & 2 & 4 & 2 & $\mathrm{IC} / \mathrm{EP}$ \\
\hline \multicolumn{6}{|l|}{ Otaridae } \\
\hline Otaria flavescens * & 73 & 243 & 130 & 75 & $\mathrm{~N} / \mathrm{MP}$ \\
\hline \multicolumn{6}{|l|}{ Phocidae } \\
\hline Hydrurga leptonyx & 0 & 1 & 0 & 0 & $\mathrm{~N} / \mathrm{MP}$ \\
\hline \multicolumn{6}{|l|}{ Balaenopteridae } \\
\hline Balaenoptera musculus & 0 & 0 & 12 & 0 & $\mathrm{EP} / \mathrm{EP}$ \\
\hline \multicolumn{6}{|l|}{ Delphinidae } \\
\hline Cephalorhynchus eutropia & 0 & 5 & 4 & 6 & $\mathrm{IC} / \mathrm{CA}$ \\
\hline Lagenorhynchus australis & 10 & 9 & 5 & 8 & $\mathrm{~N} / \mathrm{IC}$ \\
\hline Orcinus orca & 0 & 0 & 0 & 1 & $\mathrm{~N} / \mathrm{IC}$ \\
\hline
\end{tabular}

\section{DISCUSIÓN}

De la totalidad de aves marinas observadas en bahía San Pedro, se registraron cuatro especies pertenecientes al área de endemismo "Corriente de Humboldt" [i.e. pingüino de Humboldt Spheniscus humboldti (Meyen, 1834), piquero, pelícano Pelecanus thagus (Molina, 1782) y lile Phalacrocorax gaimardi (Lesson \& Garnot 1828)] y tres especies pertenecientes al área de endemismo "Zona Subantártica" [i.e. fardela negra, pingüino de Magallanes y cormorán imperial Phalacrocorax atriceps (King, 1828)] (Schlatter \& Simeone 1999), lo que sugiere que el BCNRL corresponde a una zona distribucional de interfase única para ambas zonas de endemismo. Biogeográficamente, esta zona $\left(30-41^{\circ} \mathrm{S}\right)$ es considerada como un Área Intermedia extensa (no transicional) que incluye componentes mixtos de carácter subtropical y templado-frío con prevalencia de afinidad subantártica, la cual a su vez es subdivida por tres distritos, de entre los cuales bahía San Pedro es representada por el distrito Meridional (37-41 ${ }^{\circ} \mathrm{S}$ ) (Camus 2001). Esta zona de interfase caracteriza principalmente a la Región de Los Lagos y permite con ello explicar, en parte, que esta región posea la mayor riqueza de aves marinas nidificantes y número de colonias registradas en Chile (ver Vilina et al. 2006).

La colonia mixta de pingüinos de Magallanes y Humboldt existente en el sistema de islotes de bahía San Pedro forma parte de los únicos tres registros de este tipo de colonias en Chile y el mundo. Las dos colonias restantes se ubican en la costa pacífica de la isla grande de Chiloé $\left(42^{\circ} \mathrm{S}\right)$ (Simeone et al. 2009, Simeone \& Schlatter 1998), siendo la colonia de bahía San Pedro la más septentrional, existiendo una alta probabilidad que existan más colonias mixtas al sur de Chiloé (Hiriart-Bertrand et al. 2010).

De la totalidad de mamíferos marinos presentes en bahía San Pedro, cabe destacar la presencia de 12 ejemplares de ballena azul con actividad de alimentación en la bahía, observándose a una madre y su cría, a una distancia de 4 $\mathrm{km}$ de la costa, coincidiendo con el rango de distribución costera descrito para esta especie (Hucke-Gaete et al. 2004). Este fue un registro único obtenido durante enero 2008, lo que, en función a la totalidad de observaciones realizadas en periodos estivales, indica una probabilidad del 33\% para avistar este cetáceo en bahía San Pedro. Lo anterior permite sugerir a bahía San Pedro como parte de la ruta migratoria de este gran cetáceo desde bajas latitudes hacia el sitio de mayor concentración de alimentación y crianza descrito para esta especie en el hemisferio sur [Chiloé (43-44 $\mathrm{S})$ ] (Hucke-Gaete et al. 2004).

El registro de un individuo de leopardo marino en la playa de caleta San Pedro se suma a los pocos registros conocidos para esta especie en bajas latitudes de la costa chilena, pese a la reconocida dispersión anual de algunos individuos de esta especie hacia el norte de sus zonas reproductivas ubicadas en el círculo polar antártico (Rounsevell \& Eberhard 1980). La función de acumulación de Clench indicó que el número de mamíferos marinos presentes en bahía San Pedro es de 12 especies, es decir, aún quedan por registrar más del 40\% de los mamíferos marinos en el área. Pese a ello, en este estudio se registraron cuatro especies más que el numero de mamíferos marinos observados en el área marina contigua a bahía San Pedro (Osman et al. 2008). 
El cálculo de la diversidad beta para las aves marinas avistadas durante todas las temporadas mostró un menor recambio de especies en comparación con los mamíferos marinos, con una mayor similitud en el ensamble de aves, lo cual concuerda con la relación inversa entre la similitud y la diversidad beta (Moreno 2001) e indica que las especies de aves marinas en bahía San Pedro son más constantes en el tiempo que las especies de mamíferos marinos. Por otra parte, el cálculo de la diversidad beta para los mamíferos marinos presentó un mayor recambio de especies que las aves marinas en esta bahía, señalando que durante el verano de 2007 hubo un bajo intercambio de especies. Lo que temporalmente concordó con la presencia de un evento El Niño (ENSO) en las costas de Chile, fenómeno oceanográfico al cual se le atribuyen cambios en la composición del ensamble de aves y mamíferos marinos debido principalmente a la migración de sus presas (Oedekoven et al. 2001; Ballance et al. 2006). Interacción que necesita ser estudiada a largo plazo en Chile para entender sus procesos e implicancias sobre los vertebrados marinos (Jaksic \& Fariña 2010).

De las 18 especies de aves marinas presentes en bahía San Pedro, el 33,3\% de ellas (6 especies) poseen problemas de conservación a nivel nacional y mundial. Dentro de las especies amenazadas, dos de ellas nidifican en el sistema de islotes de bahía San Pedro (i.e. pingüinos de Humboldt y Magallanes). Mientras que de las siete especies de mamíferos marinos presentes en bahía San Pedro, el 42,8\% de ellas (3 especies) poseen problemas de conservación, de las cuales una especie (i.e. chungungo) se reproduce en la bahía. Así también, la presencia en bahía San Pedro de tres especies de cetáceos (Delphinidae) considerados a nivel mundial como insuficientemente conocidos, abren la oportunidad para realizar programas de estudios que generen información ecológica sobre estas especies e incentiven el desarrollo de investigadores locales que den continuidad a dichos programas.

IMPORTANCIA PARA LA CONSERVACIÓN DE LA BIODIVERSIDAD Para el BCNRL se ha reportado la existencia de 11 loberas de lobo marino común (Osman et al. 2008; Oliva et al. 2007), de las cuales la ubicada en bahía San Pedro, según los presentes datos, es la tercera en nivel de abundancia. De los tres sitios de crianza conocidos para chungungo en el BCNRL (Córdova et al. 2009; Osman et al. 2008), bahía San Pedro se suma como el cuarto sitio descrito para la zona. Con respecto a las colonias de aves marinas, existen cinco sitios de nidificación registrados en el BCNRL (Cursach et al. 2009; Osman et al. 2008; Frere et al. 2004), de entre los cuales bahía San Pedro posee la mayor riqueza de especies nidificantes. En suma a lo anterior, la existencia de poblaciones residentes de especies amenazadas de extinción (i.e. pingüinos y chungungos) demuestran el rol fundamental que posee esta bahía para la conservación de la diversidad de aves y mamíferos marinos en el BCNRL.

La importante biodiversidad marina que posee esta bahía, junto a la buena conectividad (i.e. camino vehicular) existente con el centro urbano más cercano (i.e. Purranque), en relación al resto del BCNRL, demuestran el evidente potencial que posee bahía San Pedro para el desarrollo de actividades turísticas de intereses especiales.

Durante los últimos 10 años (1999-2009), la visita de turistas a las áreas silvestres pertenecientes al Sistema Nacional de Áreas Silvestres Protegidas por el Estado de Chile (SNASPE) ha crecido en más de un $21 \%$ [calculada mediante la fórmula $r=(\ln N-\ln N o) / t$; ver Rau (1997), a partir de los datos de CONAF. Entre los años 2004 y 20095, las áreas silvestres ubicadas en la Región de Los Lagos fueron las más visitadas $(37,8 \%)$ en todo el país [pese a solo poseer un $5,5 \%$ de la superficie total cubierta por el SNASPE (Rovira et al. 2008)], siendo el 35,9\% turistas extranjeros. Esta importante cantidad de turistas (nacionales y extranjeros) interesados en visitar los atractivos naturales de la Región de Los Lagos, denota el inminente aumento (a corto plazo) de turistas y servicios turísticos en bahía San Pedro. Esta situación podría brindar nuevos ingresos económicos para la comunidad local (i.e. comunidades Mapuche-Huilliches, pescadores artesanales y agricultores). Sin embargo, de no ser una actividad planificada y regulada, esta actividad puede generar impactos perjudiciales en el ambiente [e.g. incremento del consumo de suelo, agua y energía local, cambios en el uso de la tierra y alteración de ecosistemas, aumento de basura y aguas residuales, introducción de especies exóticas, aumento de incendios forestales, pérdida de valores tradicionales y culturales, aumento de precios que afectan a la población local, incidiendo en la pérdida de sus propiedades, comercios y servicios (Gössling 2002; Santamarta 2000)]. Es por lo tanto conveniente poner en práctica estrategias de gestión que otorguen al turismo un rol activo que minimice los impactos negativos sobre el medio natural y contribuya a la salud y viabilidad del ambiente visitado (Orams 1995). Para ello es necesaria la elaboración de un programa de turismo en conservación que incluya un programa de monitoreo a largo plazo orientado a proyectos de investigación sobre la biodiversidad presente, sus niveles de tolerancia a factores antropogénicos y sus cambios en el tiempo.

Se concluye que bahía San Pedro sustenta una importante diversidad de aves y mamíferos marinos respeto de la totalidad del BCNRL, siendo necesario aumentar el esfuerzo de estudio principalmente sobre el ensamble de mamíferos marinos. Los ensambles de aves y mamíferos marinos presentes en bahía San Pedro poseen dinámicas temporales cuyas causas deben ser investigadas. El paisaje 
actual de esta bahía exige con urgencia generar propuestas que garanticen la conservación y correcto desarrollo de esta bahía y sus habitantes.

\section{AGRADECIMIENTOS}

A la I. Municipalidad de Purranque y al Departamento de Ciencias Básicas de la Universidad de Los Lagos por financiar el trabajo de terreno de esta investigación. A los integrantes del Sindicato de Pescadores Artesanales de bahía San Pedro. A la Dirección de Investigación de la Universidad de Los Lagos por financiar los costos de esta publicación. J. Cursach, C. Tobar y J. Vilugrón agradecen el apoyo de la beca Nibaldo Bahamonde. J. Ojeda agradece a la beca de Magíster del Proyecto ICM, código P05-002 otorgada por el IEB. C.G. Suazo agradece el apoyo de CONICYT. A Daniela Haro, Dr. Alejandro Simeone y a un revisor anónimo de Gayana, por los aportes realizados a este escrito.

\section{BIBLIOGRAFÍA}

Aguayo, A., Acevedo, J. \& Vargas, R. 2006. Diversidad de mamíferos marinos en las aguas del archipiélago de Los Chonos (4339'S - 4550'S), XI región de Chile. Revista Ciencia y Tecnología del Mar 29:129-145.

Ballance, L.T., Pitman, R.L. \& Fiedler, P.C. 2006. Oceanographic influences on seabirds and cetaceans of the eastern tropical Pacific: A review. Progress in Oceanography 69:360-390.

Bibby, C., Burgess, N., Hill, D. \& Mustoe, S. 2000. Bird census techniques. Academic Press, London. 302 pp.

Camus, P. 2001. Biogeografía marina de Chile continental. Revista Chilena de Historia Natural 74:587-617.

Constantine, R. 1999. Effects of tourism on marine mammals in New Zealand. Science for Conservation 106:1173-2946. URL: http://www.doc.govt.nz/upload/documents/scienceand-technical/sfc106.pdf. Accesado: Diciembre 11, 2010.

Córdova, O., Rau, J.R., Suazo, C.G. \& Arriagada, A. 2009. Estudio comparativo de la ecología alimentaria del depredador de alto nivel trófico Lontra felina (Molina, 1782) (Carnivora: Mustelidae) en Chile. Revista de Biología Marina y Oceanografía 44:429-438.

Cursach, J., Vilugrón, J., Tobar, C., Ojeda, J., Rau, J., Oyarzún, C. \& Sото, O. 2009. Nuevos sitios de nidificación para cuatro especies de aves marinas en la provincia de Osorno, centro-sur de Chile. Boletín Chileno de Ornitología 15:1722.

Ellenberg, U., Mattern, T., Seddon, P.J. \& Luna-Jorquera, G. 2006. Physiological and reproductive consequences of human disturbance in Humboldt penguins: the need for species-specific visitor management. Biological Conservation 133:95-106.

Frere, E., Gandini, P., Ruiz, J. \& Vilina, Y. 2004. Current status and breeding distribution of Red-legged Cormorant Phalacrocorax gaimardi along the Chilean coast. Bird Conservation International 14:115-123.
Fowler, G.S. 1999. Behavioral and hormonal response on Magellanic penguins (Spheniscus magellanicus) to tourism and nest visitation. Biological Conservation 90:143-149.

Franja, 1993. Programa índice de diversidad (DIVERS.EXE) y medidas de similitud (SIMIL.EXE). Madrid.

GössLing, S. 2002. Global environmental consequences of tourism. Global Environmental Change 12:283-302.

Heltsche, J.F. \& Forrester, N.E. 1985. Statistical evaluation of the jackknife estimate of diversity when using quadrat samples. Ecology 66:107-111.

Hiriart-Bertrand, L., Simeone, A., Reyes-Arriagada, R., Riquelme, V., Pütz, K. \& Lüthi, B. 2010. Descripción de una colonia mixta de pingüino de Humboldt (Spheniscus humboldti) y de Magallanes (S. magellanicus) en isla Metalqui, Chiloé, sur de Chile. Boletín Chileno de Ornitología 16: 42-47.

Hucke-Gaete, R., Osman, L.P., Moreno, C.A., Findlay, K.P. \& LuJngBlad, D.K. 2004. Discovery of a blue whale feeding and nursing ground in southern Chile. Proceedings of the Royal Society B 271:170-173.

Hutcheson, K. 1970. A test for comparing diversities based on the Shannon formula. Journal of Theoretical Biology 29:151154.

JAKSIC, F.M. \& FARIÑA, J.M. 2010. El Niño and the birds: a resourcebased interpretation of climatic forcing in the southeastern pacific. Anales Instituto de la Patagonia 38:121-140.

Krebs, C.J. 1999. Ecological methodology. Second Edition. Addison-Wesley Educational Publishers Inc, New Jersey. 624 pp.

Moreno, C. 2001. Métodos para medir la biodiversidad. M\&T - Manuales y Tesis SEA, Zaragoza. 83 pp.

Oedekoven, C.S., Ainley, D.G.\& Spear, L.B. 2001. Variable responses of seabirds to change in marine climate: California Current, 1985-1994. Marine Ecology Progress Series 212:265-281.

Oliva, D., Sielfeld, W., Buscaglia, M, Matamala, M., Moraga, R., Pavés, H., Pérez, M.J., Schrader, D. \& Sepúlveda, M. 2007. Plan de acción para disminuir y mitigar los efectos de las interacciones del lobo marino común (Otaria flavescens) con las actividades de pesca y acuicultura de la X y XI región. Informe Final FIP N ${ }^{\circ}$ 2006-34. URL: http:// www.fip.cl/FIP/Archivos/pdf/informes/inffinal200634.pdf. Accesado: Noviembre 25, 2010.

Orams, M. 1995. Towards a more desirable form of ecotourism. Tourism Management 16:3-8.

Osman, L., Ruiz, J, Farías, A. \& CARdyn, S. 2008. Diagnóstico complementario de la avifauna y mamíferos marinos del Área Marina Costera Protegida de Múltiples Usos Lafken Mapu Lahual, región de Los Lagos. URL: http://www. conama.cl/gefmarino/1307/articles-47874_informeFinal. pdf. Accesado: Noviembre 26, 2010.

RAU, J. 1997. Factores de impacto de la Revista Chilena de Historia Natural: 1991-1995. Revista Chilena de Historia Natural 70:453-457.

Rounsevell, D. \& Eberhard, I. 1980. Leopard seals, Hydrurga leptonyx (Pinnipedia), at Macquarie Island from 1949 to 1979. Australian Wildlife Research 7: 403-415.

Rovira, J., Ortega, D., Álvarez, D. \& Molt, K. 2008. Áreas protegidas en Chile. En: Biodiversidad de Chile: Patrimonio y Desafíos (Eds. CONAMA), pp: 506-561. 
Tercera edición. Editorial Ocho Libros, Santiago.

Santamarta, J. 2000. Turismo y Medioambiente. World Watch Institute, pp: 52-55. URL: http://www.magister.com.py/ py_turistico/art.turismoymedioambiente.pdf. Accesado: Diciembre 02, 2010.

Schlatter, R. \& Simeone, A. 1999. Estado del conocimiento y conservación de las aves en mares chilenos. Estudios Oceanológicos 18:25-33.

Simeone, A. \& Schlatter, R. 1998. Threats to mixed-species colony of Spheniscus penguins in southern Chile. Colonial Waterbirds 21:418-421.

Simeone, A., Hiriart-Bertrand, L., Reyes-Arriagada, R., Halpern, M., Dubach, J., Wallace, R, Putz, K. \& Lüthi, B. 2009. Heterospecifing pairing and hybridization between wild Humboldt and Magellanic penguin in southern Chile. Condor 11:544-550.

Skewgar, E., Simeone, A. \& Boersma, P.D. 2009. Marine reserve in Chile would benefit penguins and ecoturism. Ocean \& Coastal Management 52:487-491.

Smith-Ramírez, C. 2004. The Chilean coastal range: a vanishing center of biodiversity and endemism in South American temperate rainforests. Biodiversity and Conservation 13:373-393.

Soberón, J. \& Llorente, J. 1993. The use of species accumulation functions for the prediction of species richness. Conservation Biology 7:480-488.

Tasker, M., Hope Jones, P., Dixon, T. \& Blake, B. 1984. Counting seabirds at sea from ships: a review of methods employed and a suggestion for a standardized approach. The Auk 101:567-577.

Vilina, Y., Cofré, H. \& Pizarro, C. 2006. Reporte final aves acuáticas de Chile. Waterbird Conservation of the Americas, BirdLife International. URL: http://www. birdlife.org/action/science/species/waterbirds/waterbirds pdf/waterbirds_report_chile_2006.pdf. Accesado: Abril 19, 2011

Yorio, P., Frere, E., Gandini, P. \& Schiavini, A. 2001. Tourism and recreation at seabird breeding sites in Patagonia, Argentina: current concerns and future prospects. Bird Conservation International 11:231-245.

ZAR, J.H. 1974. Biostatistical analysis. Prentice-Hall Inc, New Yersey. 620 pp.

Recibido: 22.12 .10

Aceptado: 20.05.11 